KANAZAWA-98/07

HUB-EP-98/64

ITEP $49 / 98$

\title{
Background Configurations, Confinement and Deconfinement on a Lattice with BPS Monopole Boundary Conditions
}

\author{
E.-M. Ilgenfritz ${ }^{1}$, S.V. Molodtsov², M. Müller-Preussker ${ }^{3}$, \\ and A.I. Veselov ${ }^{2}$ \\ 1 Institute for Theoretical Physics, University of Kanazawa, Japan \\ 2 Institute of Theoretical and Experimental Physics, Moscow, Russia \\ 3 Institut für Physik, Humboldt-Universität zu Berlin, Germany
}

September 25, 1998

\begin{abstract}
Finite temperature $S U(2)$ lattice gauge theory is investigated in a $3 D$ cubic box with fixed boundary conditions provided by a discretized, static BPS monopole solution with varying core scale $\mu$. Using heating and cooling techniques we establish that for discrete $\mu$-values stable classical solutions either of self-dual or of pure magnetic type exist inside the box. Having switched on quantum fluctuations we compute the Polyakov line and other local operators. For different $\mu$ and at varying temperatures near the deconfinement transition we study the influence of the boundary condition on the vacuum inside the box. In contrast to the pure magnetic background field case, for the self-dual one we observe confinement even for temperatures quite far above the critical one.
\end{abstract}




\section{Introduction}

Quark confinement is one of the most intriguing problems in QCD. There are several appealing analogies to SQCD and supergravity which are helpful to guide our intuition. Nevertheless, a final explanation of the mechanism in terms of structures of (non-supersymmetric) QCD or pure Yang-Mills theory itself is still missing. More precisely, it is confinement at weak coupling, when the lattice spacing is well below the presumably relevant structures accomplishing this mechanism, that we need to explain. Therefore a closer investigation of various semi-classical configurations in the context of lattice simulations in different phases seems to be necessary.

The first example of an extended excitation which is looked for, nowadays massively, in Monte Carlo generated gauge field configurations is the instanton [1]. It is not completely clear at present to what extent their size (actually their distribution of sizes) is influenced by the detection algorithm itself [2, 3], but there is no doubt that this scale can be and perhaps has been already decoupled from the lattice spacing. I

In a recent publication lattice measurements of the field strength correlator at zero temperature have been confronted with semi-analytic results of the semi-classically motivated instanton liquid approximation [5]. The correlation length and strength of the correlation at intermediate distances can be explained within the standard parameters characterizing the instanton liquid. However, the change of the field strength correlators at the deconfining transition cannot be explained in an model based exclusively on self-dual or anti-self-dual configurations [6].

According to current folklore, instantons are not related to confinement at all. If they are related, we have still to identify the interactions and correlations in the instanton liquid which would make the corresponding sample fields confining. Traditionally, in continuum models, instantons are the relevant configurations to explain spontaneous chiral symmetry breaking and are needed to solve the $U_{A}(1)$ problem [1]. Without the inclusion of (short range) instanton correlations one is not able to unterstand the temperature dependence of the chiral condensates and the restoration of chiral symmetry at $T_{c h}$ (for a schematic model, see Ref. [7]). An indirect role for confinement is attributed to instantons because of local correlations between monopoles and instantons [8].

Monopoles are the agents of another model that has been first formulated in the continuum in order to explain confinement: the dual superconductor scenario invented by t'Hooft and Mandelstam [9]. It assumes condensation of Abelian monopoles and views confinement as a dual Meissner effect. That this scenario really takes place has been demonstrated for Abelian compact and non-Abelian lattice gauge theories by numerical simulations [10, 11]. In the latter case Abelian monopoles appear as singularities of the gauge fixing procedure and are identified as point-like conserved currents (DeGrand-Toussaint monopoles [12]) in various gauges after Abelian projection. These monopoles have a quantized magnetic charge but no natural size which would be analogous to the instanton size and could point to some confinement scale and corresponding QCD coupling. In the very first attempt it was tried

\footnotetext{
${ }^{1}$ Perfect actions, however, are trying to go the opposite way [4]. At the cost of a complicated but still practically feasible action one wants to be able to keep the discretization scale near to the dynamically important scale(s) whereas good continuum features can be obtained within bigger volumes in affordable computer time.
} 
to prove that their density possesses a continuum limit [13]. But the density is strongly affected by short-range lattice artefacts. There have been methods developed to remove these artefacts (monopole blocking to obtain an infrared effective action for thick monopoles [14], renormalization group motivated smoothing of configurations which reduces point-like Abelian monopole currents [15).

There is one model for an extended, particle-like monopole which could be confronted with first-principle lattice Monte Carlo configurations. This is the t'Hooft-Polyakov (HP) [16] or - as the limiting case - the Bogomol'nyi-Prasad-Sommerfield (BPS) monopole [17]. It is taken from the Georgi-Glashow model and re-interpreted as a static configuration in pure gauge theory identifying the adjoint Higgs field with the $4^{\text {th }}$ component of the vector potential. As a solution of the classical non-Abelian field equations the BPS monopole satisfies the self-duality condition and carries electric charge. This is the reason why in pure gauge theory we want to call the BPS monopole solution also dyon. I

The present paper represents an attempt to examine isolated configurations of this kind under the influence of quantum fluctuations. A first investigation in this spirit was reported by Smit and van der Sijs [19]. A particular feature in our present work (similar as in [19]) is the possibility to select the semi-classical background field by applying specific boundary conditions fixing the tangential vector potential at the surface of a spatial box. The main emphasis in the work of our precedessors was to select the coupling (or length scale) at which these monopoles would condense at zero physical temperature (and to determine the mass of the quantum monopole) in the spirit of Ref. [20]. Here, in contrast, we want first to elaborate on the question which solutions of the lattice equations of motion are compatible with the boundary conditions. Only if they are invariably recovered from heating and cooling cycles we can speak of unique background configurations. We will discover that, although the boundary conditions are originally taken from a self-dual BPS configuration, depending on discrete values of the size parameter $\mu^{-1}$, these solutions can have either pure magnetic monopole or dyonic (self-dual) character. For simplicity, let us call them in the first case $H P$ monopole (because of the suppressed electric components), in the second one BPS monopole or simply dyon. Our main interest is to investigate for the finite temperature case how usual indicators of the deconfinement phase transition (average Polyakov line, distribution of Polyakov lines) are modified by the presence of the different kinds of background fields. For this purpose we have to consider these observables locally, sufficiently far from the 'cold walls' of the box.

Our main result will be that the self-dual BPS-like (dyon) background fields - in contrast to the pure magnetic HP monopole ones - strongly support confinement. We shall show that a dyon invironment keeps the Yang-Mills theory in the confinement state even for temperatures $T>T_{c}$.

From the investigation of the field strength correlator [5] it is known that self-dual semiclassical configurations seem to be essential in the confinement phase but only there. Investigations of Wilson loops in the classical dyon background have shown that dyons give rise, at short distances, to a weak but confining quark-antiquark force of harmonic oscillator type ('super-confinement') [21]. 3 Notice that this is not the confining force at long distances

\footnotetext{
${ }^{2}$ One should not mistake this dyon solution with the Julia-Zee dyon in the Georgi-Glashow model 18$]$.

${ }^{3}$ Similar observations have been made for the short range potential in the field of an instanton [22] and for more generic self-dual fields [23].
} 
described by the string tension or the magnetic confinement [24] for space-like Wilson loops at high temperature. With respect to the latter, a dense gas of magnetic monopoles at high temperatures could explain it.

The paper is organized as follows. In chapter 2 we show how to discretize a continuum BPS monopole (dyon) solution and how the classical lattice configurations compatible with dyonic boundary conditions can be classified interpolating between lattice HP monopoles and dyons. In chapter 3 we report on Monte Carlo simulations performed with fixed open spatial boundary conditions corresponding to HP monopole or dyon background fields at finite temperature. We measure the profile of the action density and Polyakov line inside the box and establish the existence or absence of confinement. Chapter 4 contains the conclusions.

\section{Classical Solutions with Dyon Boundary Conditions}

First let us discuss classical field configurations in a finite volume with fixed spatial boundary conditions. We consider a hypercubic lattice of size $N_{s}^{3} \times N_{t}$ with periodic boundary conditions in the imaginary time direction (finite temperature $T=1 /\left(a \cdot N_{t}\right)$ ) and open boundary conditions in the three-space. The latter will be specified such that links being normal to the boundary and pointing outwards are inactive, i. e. cannot contribute to the action. Link variables tangential to the boundaries will be fixed to classical values given by a BPS dyon configuration as defined in the following.

We discretize a single BPS dyon field being a static solution of the Euclidean Yang-Mills equations of motion. Its center be fixed at $x_{1}=x_{2}=x_{3}=\left(N_{s}+1\right) / 2$. For the lattice spacing $a=1$ is assumed. On each time slice $t \equiv x_{4}$ all active link variables $U_{\nu}(\vec{x}, t) \in S U(2)$, $\nu=1, . ., 4$, are represented as follows:

$$
\begin{aligned}
U_{k}(\vec{x}, t) & =\exp \left(\frac{-i}{2} \sigma_{j} \varepsilon_{j k l} x_{l} \int_{0}^{1} d s \frac{1-K(\mu r(s))}{r^{2}(s)}\right) \\
U_{4}(\vec{x}, t) & =\exp \left(\frac{-i}{2} \sigma_{j} x_{j} \frac{H(\mu r)}{r}\right), \quad j, k, l=1,2,3,
\end{aligned}
$$

with $r(s)=\sqrt{\left(x_{k}+s\right)^{2}+\sum_{j \neq k} x_{j}^{2}}, \quad r(0)=r$. For the BPS dyon solution we have explicitely

$$
\begin{aligned}
K(\mu r) & =\frac{\mu r}{\sinh (\mu r)} \\
H(\mu r) & =\mu r \frac{\cosh (\mu r)}{\sinh (\mu r)}-1 .
\end{aligned}
$$

$\mu$ denotes the inverse core size of the dyon to be fixed lateron. The replacement $\mu \rightarrow-\mu$ transforms the dyon into an anti-dyon. Note that in the limit $r \rightarrow \infty$

$$
\frac{1}{r} H(\mu r) \sim \mu-\frac{1}{r}-O(\exp (-\mu r)) .
$$


For comparison, the asymptotic behaviour of an HP pure monopole solution (which classically would be related to a sufficiently strong non-vanishing Higgs potential and in the quantum case of pure $S U(2)$ gauge theory could be due to a dynamical screening of the electric field components) is determined by

$$
\frac{1}{r} H(\mu r) \sim \mu-O(\exp (-\mu r))
$$

It is immediately clear that at semi-asymptotic distances one cannot distinguish between a classical BPS dyon and a HP monopole if one replaces

$$
\mu_{\text {dyon }}-\frac{1}{r} \rightarrow \mu_{\text {monopole }}
$$

As has been shown by Smit and van der Sijs [19] the dyon or monopole fields at the boundary have some further interesting properties. Additionally to the Abelian gauge symmetry with respect to transformations

$$
\Omega(\vec{x}, t)=\exp \left(\frac{i \omega}{2} \sigma_{j} x_{j}\right)
$$

( $\omega=$ const. $)$ the boundary fields periodic in $t$ exhibit a combined symmetry with respect to the gauge transformation

$$
\Omega(\vec{x}, t)=\exp \left(i \pi n \frac{t}{N_{t}} \sigma_{j} x_{j}\right)
$$

and the shift

$$
\mu \rightarrow \mu+\frac{2 \pi n}{N_{t}}, \quad n=1,2, \ldots
$$

Both the shifts (5) and (8) can be combined and let us expect a periodic behaviour in $\mu$ which allows to create boundary conditions compatible with a BPS dyon as well as with a HP monopole. As far as on the hypercubic lattice we have no cylindric symmetry at the boundary we have to replace $r(\vec{x})$ by an effective $R_{e f f}$, for which we adopt the value $R_{e f f} \simeq 1.13 \cdot \frac{1}{2}\left(N_{s}-1\right)$ as given in [19]. In what follows we shall parametrize the boundary values by

$$
\mu^{\prime}=\left(\mu-\frac{1}{R_{e f f}}\right) \cdot \frac{N_{t}}{2 \pi}
$$

instead of $\mu$.

In general, one cannot expect the lattice discretized dyon to be a solution of the lattice equations of motion. In order to find such a solution corresponding to the dyon boundary conditions we keep the tangential boundary links fixed at the classical values given by (1), 2). For varying $\mu$ or $\mu^{\prime}$, respectively, the other links inside the box (for all time slices) are exposed to repeated cycles of Monte Carlo heating followed by cooling. These links possess the form (11, 2) only as start values. We wanted to find out, what lattice fields, respecting the boundary conditions augmented by periodicity in time, have minimal action. It turned out that there exist several local minima of action for $\mu^{\prime}$ sufficiently big. The configurations with lowest action show the interesting $\mu^{\prime}$-dependence anticipated above. 
In order to characterize the lattice field configurations obtained by cooling after heating the discretized BPS dyon we will use the full plaquette action

$$
S=\beta \sum_{x, \mu<\nu}\left(1-\frac{1}{2} \operatorname{Tr} U_{\mu, \nu}(x)\right)=E^{2}+B^{2}, \quad \beta \equiv 4 / g^{2}
$$

with its magnetic part $B^{2}$ coming from the sum of space-space plaquette contributions and its electric part $E^{2}$ obtained as the sum of time-like plaquettes. Fig. 1 shows the full action per timeslice of the classical continuum BPS dyon, lattice discretized according to (11, 2), in dependence on the parameter $\mu^{\prime}$ (solid line). The action values are given in units of $4 \pi /\left(a g^{2}\right)$. The lattice size is $12^{3} \times 4$. Obviously, for $\mu^{\prime} \simeq 2$ the dyon core parameter becomes sufficiently large such that this dyon completely fits into the lattice box.

The data points in Fig. 1 show the global or local minima, respectively, found for the full action (asterisks) after cooling down the Monte Carlo heated configurations. Only in the range $0 \leq \mu^{\prime} \leq 0.5$ (i.e. for a sufficiently smooth discretized original dyon field (11, 2)) the action is practically reproduced. For larger $\mu^{\prime}$-values (less than 1.5) the solutions of the lattice equations of motion deviate from the original discretized dyon but are still unique in the sense that they are restored after repeated heating and cooling. These solutions exhibit the periodicity in $\mu^{\prime}$ as discussed before. For $\mu^{\prime} \geq 1.5$ we have seen also other branches of solutions with higher action than the lowest possible one which are metastable under cooling.

In addition to the full action, the magnetic (open squares) and electric contributions (full dots) to the action are shown separately. The most interesting for us are the states reached at half-integer and integer $\mu^{\prime}$, respectively. For $\mu^{\prime} \simeq 0.5,1.5, \cdots$ we reproduce a BPS dyon state identical to the original one at $\mu^{\prime}=0.5$ with equal electric and magnetic contributions to the action. For $\mu^{\prime} \simeq 1.0,2.0, \cdots$ only the magnetic part of the action survives. That means that we have obtained purely magnetic, HP-like monopoles.

We convinced ourselves that we have obtained static solutions with localized action and topological charge (the latter for the BPS dyon only). As an example we show the action density profile for the case $\mu^{\prime}=1.0$ being a pure HP-monopole (see Fig. 2).

In order to see what seems to be important of the boundary fields for the classes of lattice solutions we have found and for the classification below of the quantum fields on these background fields we compute the Polyakov line. Fig. 3 shows the Polyakov line averaged over spatial points $\vec{x}$ on the boundary, where the fields are kept at classical (discretized) BPS dyon values, as a function of $\mu^{\prime}$. We see the periodicity in $\mu^{\prime}$ again. For integer values $\mu^{\prime}$, where inside the box HP monopoles are supported as classical solutions, we have $<L>\simeq \pm 1$. On the contrary for half-integer $\mu^{\prime}$ providing BPS dyon solutions $<L>\simeq 0$. (Small deviations of the positions of the minima from 0 and \pm 1 are due to an uncomplete optimization of the $R_{e f f}$-value on $N_{s}$ in the definition of $\mu^{\prime}$.)

\section{Quantum Fields with Dyon Boundary Conditions}

In the following we want to investigate quantum fields with fixed spatial boundary conditions as given by the ansatz (1, 2). We call the latter simply dyon boundary conditions. But we should keep in mind that these boundary conditions for appropriate values of $\mu^{\prime}$ are 
compatible to classical BPS dyon or HP monopole solutions of the lattice equations of motion. We emphasize that these solutions are stable. This provides the opportunity to study a real semi-classical situation with a unique background field parametrized by $\mu^{\prime}$.

All our present work refers to $S U(2)$ pure gauge theory on a thermal (non-symmetric) lattice as a function of $\beta$ and $\mu^{\prime}$. The simulations have been done with a standard Monte Carlo Metropolis algorithm. For comparison, we have also done simulations at the same $\beta$ values on the same lattices with periodic boundary conditions. For the simulations, the lattice size has been $16^{3} \times 4$. Remember that for $N_{t}=4$ the deconfinement phase transition occurs at $\beta=\beta_{c} \simeq 2.29$ [25. In the following we will mainly consider two typical cases: $\beta=2.2$, characteristic for the confinement phase, and $\beta=2.4$, for the deconfinement phase.

In order to estimate the range of influence of the dyon boundary condition on the quantum fields we computed different local observables for various $\mu^{\prime}$ as a function of the (minimal) distance $d$ from the lattice site to the boundary. Figs. 4 and 5 show the plaquette contributions to the magnetic and electric part of the action averaged over all plaquettes with a given distance $d$. The situation for both typical $\beta$-values looks quite similar. Inside the box we obtain plateau values. The range of the plateau seems to define a core size of 'normal' quantum fluctuations. We shall investigate the properties of the quantum fields inside the core in detail. There at least no difference is seen between magnetic and electric contributions to the action.

We have computed the average Polyakov line as a function of $d$, too. This quantity is able to tell more about the influence of the type of boundary condition on the quantum fields in the interior of the box. The results are plotted in Figs. 6 and 7, respectively. Even inside the core established before (roughly at $d \geq 3$ ) the behaviour of the Polyakov line strongly depends on the boundary conditions compatible either with BPS dyon or with HP monopole background fields. For integer $\mu^{\prime}$ (HP monopole) in both the confinement and the deconfinement phase the results are compatible with the one obtained for periodic boundary conditions at the same $\beta$. That means that the interior of the lattice is in the phase corresponding to the $\beta$ value. For $\beta=2.2$, in the presence of monopole boundary conditions, the small deviation (in the center of the lattice) from the expected zero is only due to the maximal violation of the $Z(2)$ invariance right on the spatial boundary (compare with Fig. 3).

For $\beta=2.4$ (Fig. 7) we show the average Polyakov line for periodic boundary conditions in a symmetrized way. In fact, during our relatively short simulation runs of typically $O(1000)$ configurations with 50 empty sweeps we did not observe any tunneling between the $Z(2)$-symmetric states. In the presence of monopole boundary conditions, going deeper into the lattice the locally averaged Polyakov line approaches the two $Z(2)$-symmetric values. On the contrary, for half-integer $\mu^{\prime}$ (BPS dyon) the average Polyakov line remains zero throughout the whole lattice when passing the deconfinement transition. At $\beta=2.4$ inside the core we clearly get $|<L>|<0.1$. These observations are supported by the corresponding histograms of local Polyakov line values measured at distance $d=5$ from the boundary which are shown in Figs. 8 and 9 for $\beta=2.2$ and 2.4, respectively.

In the confinement phase, the histograms corresponding to the monopole b.c. are displaced representing entirely the effect of the finite correlation length of the Polyakov line, while the histogram for the dyonic boundary conditions coincides with the 'normal' one (for periodic boundary conditions). In the deconfinement phase the normal histogram (with- 
out tunneling as in our case) is reproduced with monopole boundary conditions at distance $d=5$, while the histogram for dyonic boundary conditions is almost symmetric, similar to the lower $\beta$-value. We conclude that, while the HP monopole b.c. are compatible also with the confinement phase, a BPS dyon background inside the finite box keeps the system in the confinement state even for temperatures above $T_{c}$. But as we have seen for smaller $N_{t}$ or larger $\beta$, this does not persist at arbitrarily high temperature. We checked this for $N_{t}=2, \quad \beta=2.4$ (compare Fig. 10) and $N_{t}=4, \quad \beta=2.7$. In both cases of boundary conditions we have obtained Polyakov averages inside the corresponding core regions, being compatible with the case of periodic boundaries, i.e. deconfinement states. Therefore, at larger temperatures deconfinement is restored inside the box in spite of the dyon boundary condition.

\section{Conclusions}

We have investigated pure $S U(2)$ lattice gauge theory at finite temperatures. We have imposed fixed spatial boundary conditions defined by a discretized static Bogomol'nyi-PrasadSommerfield monopole or dyon solution of the continuum Yang-Mills field equations. By minimizing the lattice action we have found stable field configurations which solve the lattice equations of motion. As already reported by Smit and van der Sijs [19 these configurations can be self-dual BPS-like dyon or pure magnetic HP-like monopole solutions. They occur periodically in terms of the core size of the original dyon as fixed at the spatial boundary.

As far as these configurations are really stable, i.e. repeated cycles of Monte Carlo heating and subsequent cooling provide always the same result, we are in a position to simulate the quantum theory in well-defined classical background fields.

We have used this environment to find out, how dyon-like or purely magnetic, monopolelike background fields influence the finite temperature quantum fields. Our main observation was that in the dyon-case the fields inside the box are kept in the confinement state even for higher temperatures, which in the standard case of periodic boundary conditions cause deconfinement. The Polyakov line as the main order parameter for the deconfinement transition turned out to be zero remarkably stable inside the whole $3 D$ volume considered. However, a further increase of the temperature finally restored the deconfinement inside the $3 D$ box. The phenomenon reported here might be called delay of confinement evaporation due to dyon boundary conditions.

We would like to interprete our findings such that the confinement phenomenon is stronger related to self-dual semi-classical objects like BPS dyons than to a pure magnetic HP monopole background. On the other hand, deconfinement, when described semi-classically, requires background fields which are not (anti-) self-dual. The final loss of confinement above $\beta=2.7$ means breakdown of semi-classical approximation. This supports the view developed in recent papers, where the correlation between instantons and monopoles has been investigated in detail on the lattice (see [8]). The idea that dyons might provide confinement within a semi-classical framework has been put forward also within the continuum approach 21].

Our results were obtained for quite small lattice sizes. We are going to check them deeper in the continuum limit and for physically larger box volumes. 


\section{Acknowledgements}

The authors are grateful to B.V. Martemyanov, A. van der Sijs, Yu.A. Simonov, J. Smit and M.I. Polikarpov for useful discussions. This work was partly supported by RFBR grants N 97-02-17491 and N 96-02-17230, INTAS-RFBR 95-0681 and INTAS 96-370. The authors acknowledge also the support by the joint DFG-RFFI grant 436 RUS 113/309/0 (R) or RFBR-DFG grant N 96-02-00088 G. M. M.-P. acknowledges partial support by the EC TMR network FMRX-CT97-0122.

\section{References}

[1] For recent reviews see

E. V. Shuryak, Rev. Mod. Phys. 65 (1993) 1;

D. Dyakonov, Prog. Part. Nucl. Phys. 36 (1996) 1;

T. Schäfer and E. V. Shuryak, Rev. Mod. Phys. 70 (1998) 323.

[2] E.-M. Ilgenfritz, M. L. Laursen, M. Müller-Preussker, G. Schierholz, and H. Schiller, Nucl. Phys. B268 (1986) 693;

M. Teper, Phys. Lett. B171 (1986) 86;

M. I. Polikarpov and A. I. Veselov, Nucl. Phys. B297 (1988) 34;

P. de Forcrand, M. Garcia Perez, and I.-O. Stamatescu, Nucl. Phys. B499 (1997) 409.

[3] J. W. Negele, Plenary talk given at 16th International Symposium on Lattice Field Theory (LATTICE 98), Boulder, CO, 13-18 Jul 1998, e-print archive hep-lat/9810053.

[4] See P. Hasenfratz, e-print archive hep-lat/9803027 and references given therein.

[5] E.-M. Ilgenfritz, B. V. Martemyanov, S. V. Molodtsov, M. Müller-Preussker, and Yu. A. Simonov, Phys. Rev. D58 (1998) 114508.

[6] E.-M. Ilgenfritz, B. V. Martemyanov, and M. Müller-Preussker, in preparation.

[7] E.-M. Ilgenfritz and E. V. Shuryak, Phys. Lett. B325 (1994) 263.

[8] M. N. Chernodub and F. V. Gubarev, JETP Lett. 62 (1995) 100;

V. G. Bornyakov and G. Schierholz, Phys. Lett. B384 (1996) 190;

S. Thurner, M. Feurstein, H. Markum, and W. Sakuler, Phys. Rev. D54 (1996) 3457;

R. C. Brower, K. N. Orginos, and C.-I. Tan, Phys. Rev. D55 (1997) 6313.

[9] G. 't Hooft, in High Energy Physics, Proc. EPS Intern. Conf., ed. A.Zichichi, 1976; Nucl. Phys. B190 (1981) 455 ;

S. Mandelstam, Phys. Rep. 23 (1976) 245.

[10] T. Banks, R. Myerson, and J. Kogut, Nucl. Phys. B129 (1977) 493;

A. S. Kronfeld, G. Schierholz, and U.-J. Wiese, Nucl. Phys. B293 (1987) 461;

A. S. Kronfeld, M. L. Laursen, G. Schierholz, and U.-J. Wiese, Phys. Lett. B198 (1987) 516 ;

T. Suzuki and I. Yotsuyanagi, Phys. Rev. D42 (1990) 4257; 
G. S. Bali, V. Bornyakov, M. Müller-Preussker, and K. Schilling, Phys. Rev. D54 (1990) 2863.

[11] L. Del Debbio, A. Di Giacomo, G. Paffuti, and P. Pieri, Phys. Lett. B355 (1995) 255;

A. I. Veselov, M. I. Polikarpov, and M. N. Chernodub, JETP Lett. 63 (1996) 411;

M. N. Chernodub, M. I. Polikarpov, and A. I. Veselov, Phys. Lett. B399 (1997) 267.

[12] T. A. DeGrand and D. Toussaint, Phys. Rev. D22 (1980) 2478.

[13] V. G. Bornyakov, E.-M. Ilgenfritz, M. L. Laursen, V. K. Mitrjushkin, M. MüllerPreussker, A. J. van der Sijs, and A. M. Zadorozhny, Phys. Lett. B261 (1991) 116.

[14] N. Arasaki, S. Ejiri, S. Kitahara, Y. Matsubara, and T. Suzuki, Phys. Lett. B395 (1997) 275 ;

S. Kato, N. Nakamura, T. Suzuki, and S. Kitahara, Nucl. Phys. B520 (1998) 323.

[15] E.-M. Ilgenfritz, M. Feurstein, M. Müller-Preussker, and S. Thurner, Nucl. Phys. B511 (1998) 421;

E.-M. Ilgenfritz, H. Markum, M. Müller-Preussker, and S. Thurner, Phys. Rev. D58 (1998) 094502.

[16] G. 't Hooft, Nucl. Phys. B79 (1974) 276;

A. M. Polyakov, JETP Letters 20 (1974) 276.

[17] M. K. Prasad and C. M. Sommerfield, Phys. Rev. Lett. 35 (1975) 760;

E. B. Bogomol'nyi, Sov. J. Nucl. Phys. 24 (1976) 449.

[18] B. Julia and A. Zee, Phys. Rev. D11 (1975) 2227.

[19] J. Smit and A. van der Sijs, Nucl. Phys. B422 (1994) 349.

[20] J. Smit and A. van der Sijs, Nucl. Phys. B355 (1991) 603.

[21] Yu. A. Simonov, Yad. Fiz. 43 (1985) 557;

B. V. Martemyanov, S. V. Molodtsov, Yu. A. Simonov, and A. I. Veselov, Pis'ma Zh. Eksp. Teor. Fiz. 62 (1995) 695.

[22] C. G. Callan, Jr., R. Dashen, D. J. Gross, F. Wilczek, and A. Zee, Phys. Rev. D18 (1978) 343.

[23] G. V. Efimov and S. N. Nedelko, Eur. Phys. J. C1 (1998) 343;

G. V. Efimov, A. C. Kalloniatis, and S. N. Nedelko, e-print archive hep-ph/9806165.

[24] Yu. A. Simonov, JETP Lett. 55 (1992) 605; Yad. Fiz. 58 (1995) 357; e-print archive hep-ph/9311216.

[25] J. Engels, J. Fingberg, and M. Weber, Nucl. Phys. B 332 (1990) 737. 


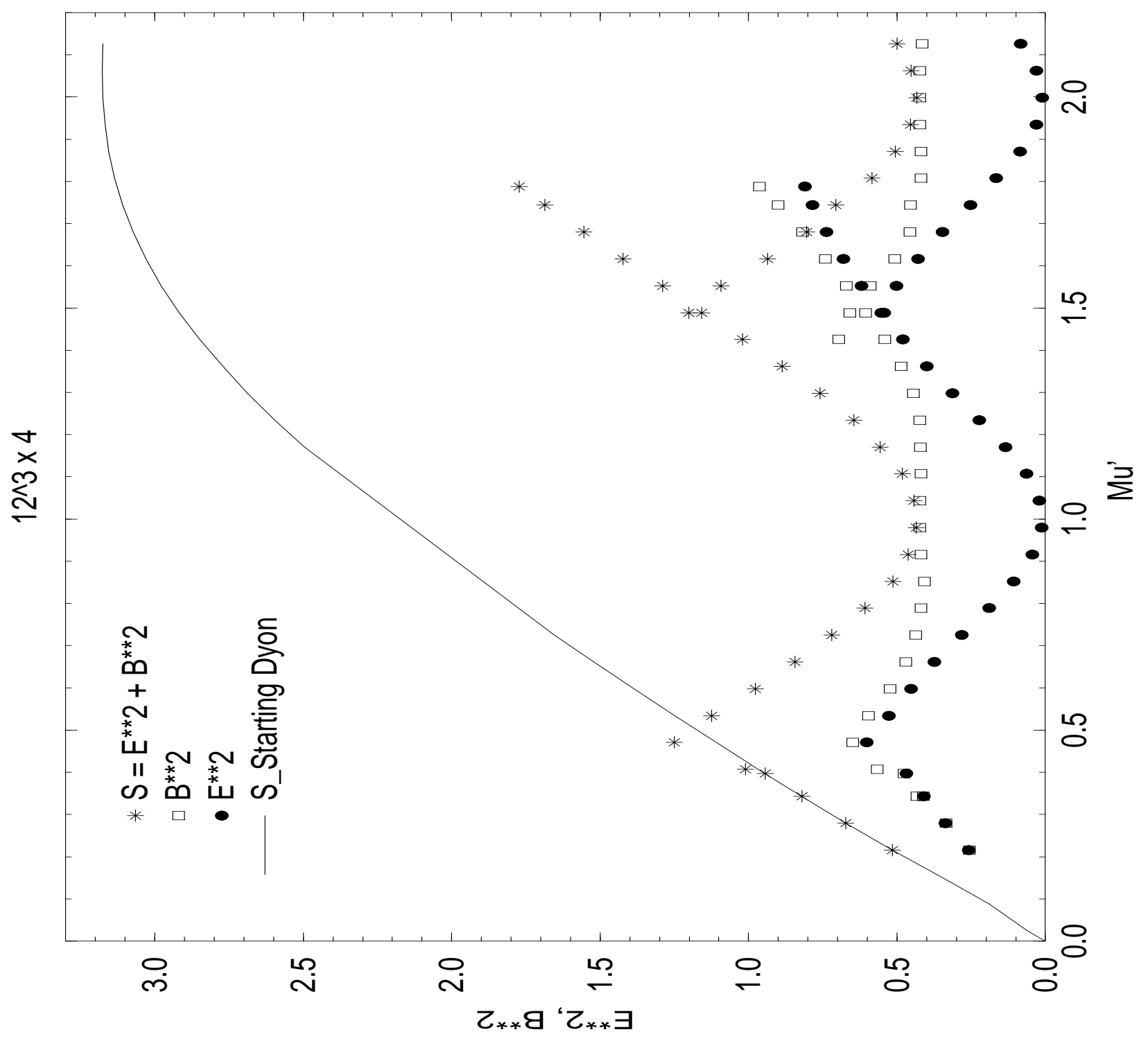

Figure 1: Total action, magnetic and electric part of the action for possible classical solutions of the lattice field equations with dyon boundary conditions as explained in the text. 


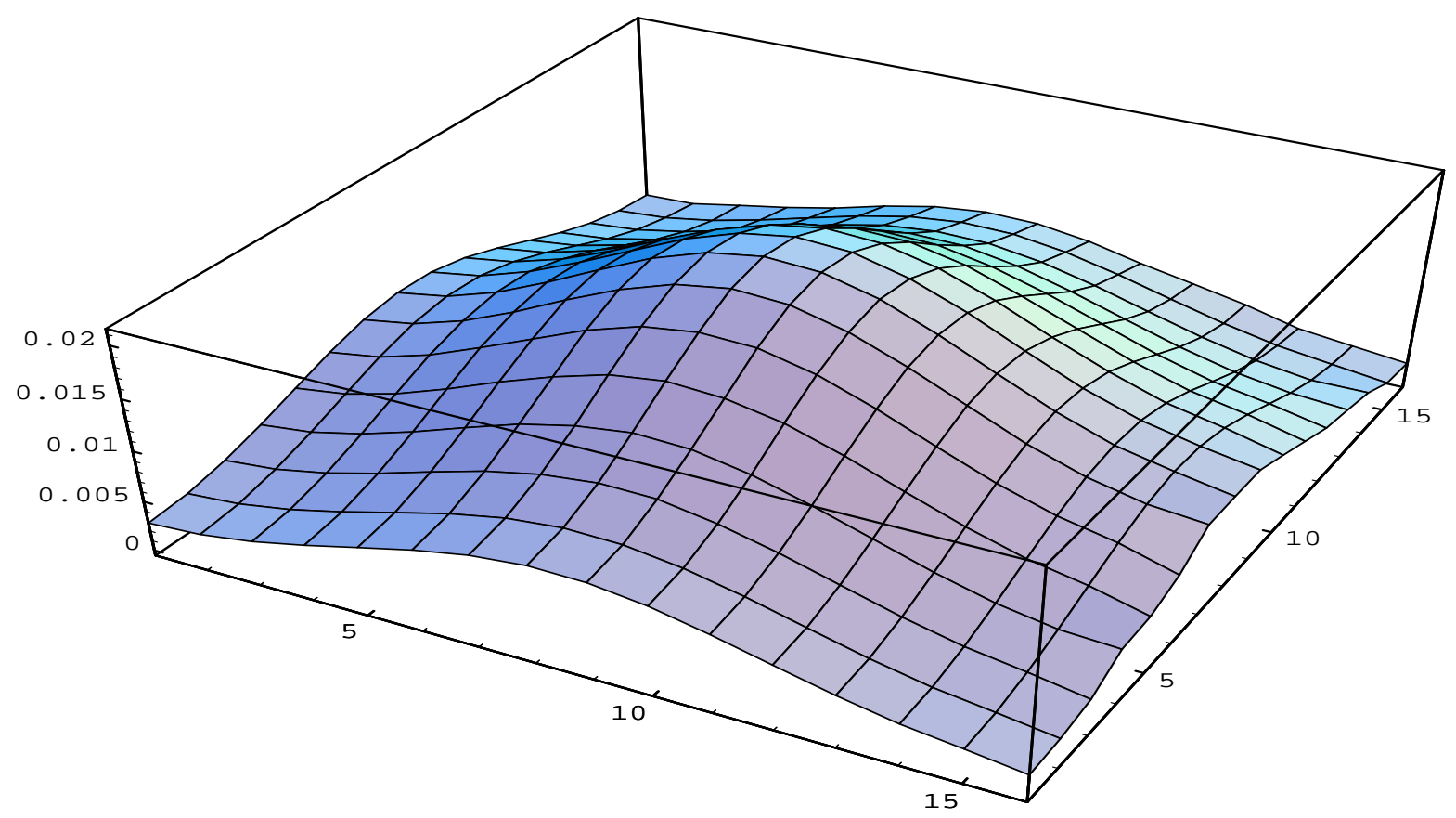

Figure 2: Action density profile of the classical solution of the lattice equations of motion for $\mu^{\prime}=1.0$ (pure magnetic monopole) as a function of $y=1, \cdots, 16$ and $z=1, \cdots, 16$. 


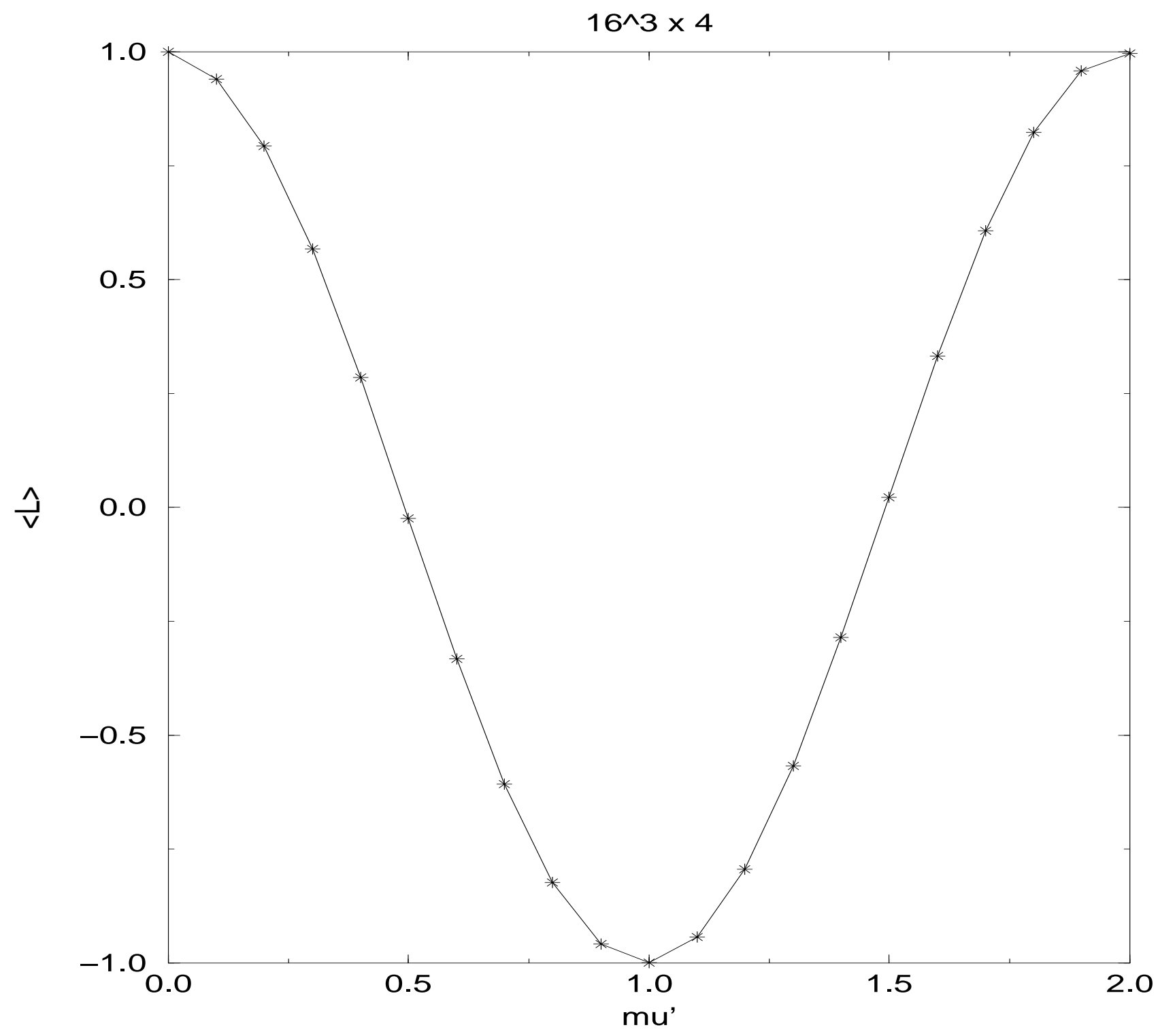

Figure 3: Polyakov line operator computed for the classical BPS configuration at the boundary of the $3 \mathrm{D}$ box for varying parameter $\mu^{\prime}$. 


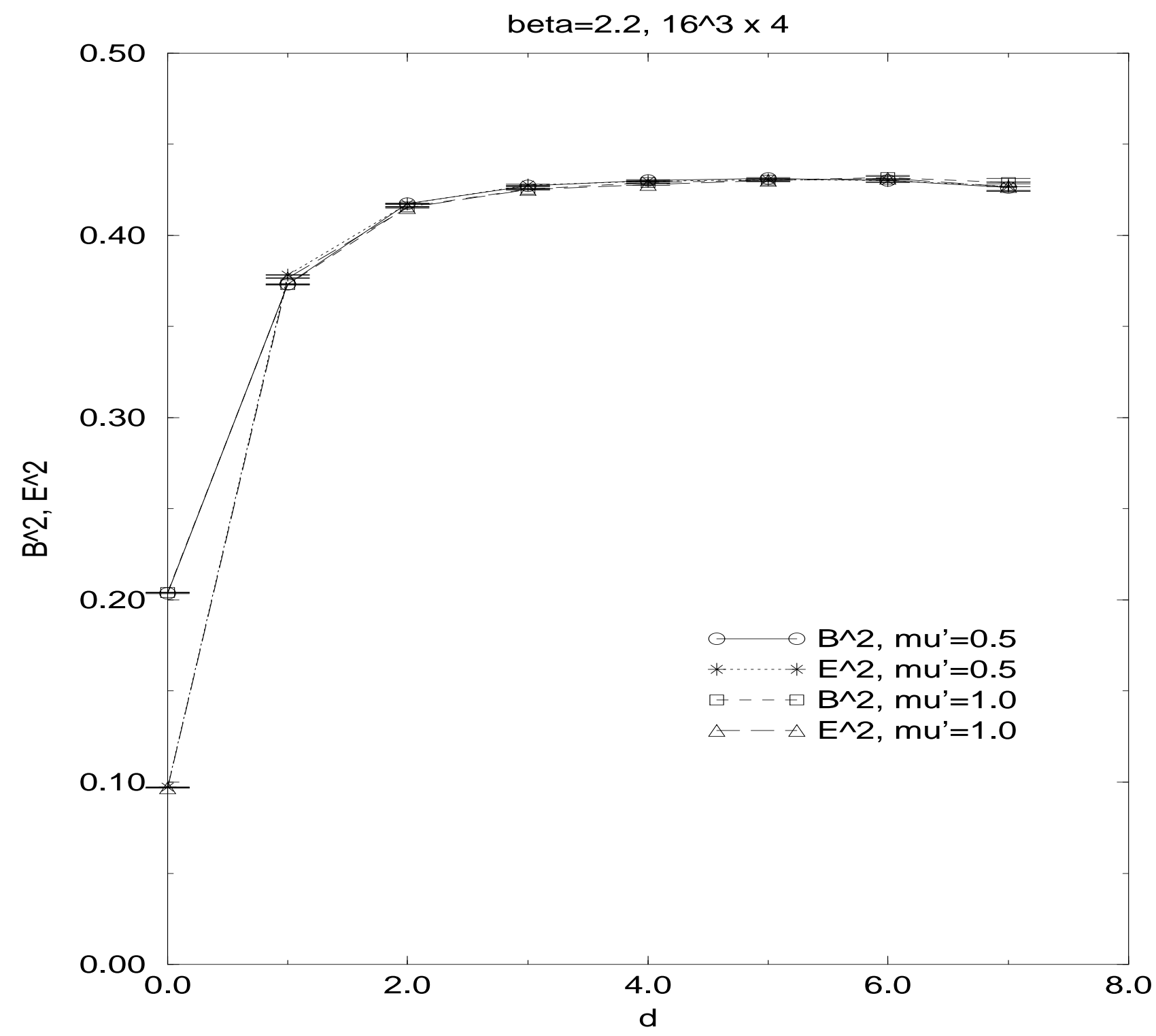

Figure 4: Magnetic and electric contributions to the full action from plaquettes with distance $d$ from the boundary in the dyon $\left(\mu^{\prime}=0.5\right)$ and in the pure monopole case $\left(\mu^{\prime}=1.0\right)$ for $\beta=2.2$ (confinement). 


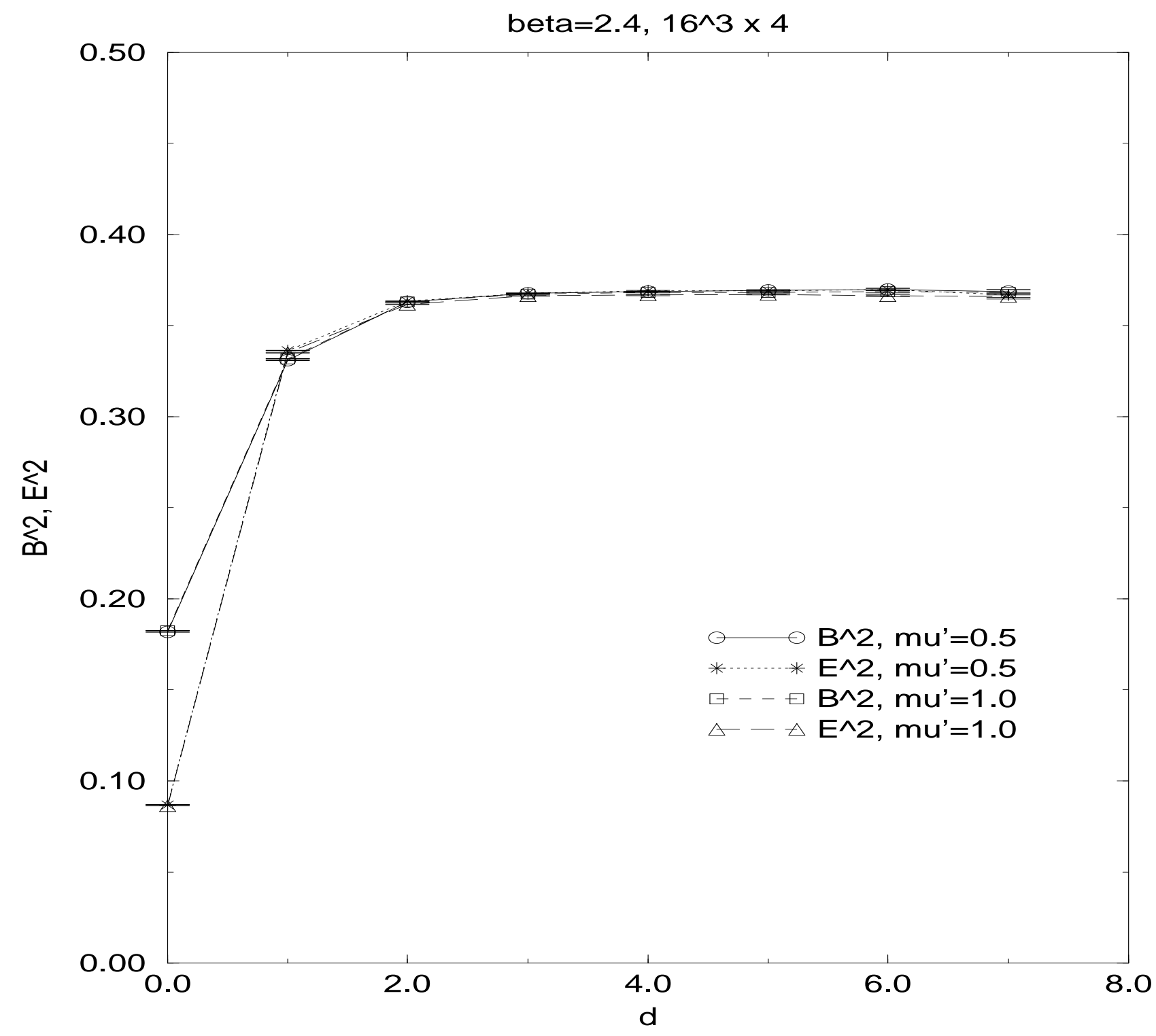

Figure 5: As in Fig. 4, but for $\beta=2.4$ (deconfinement). 


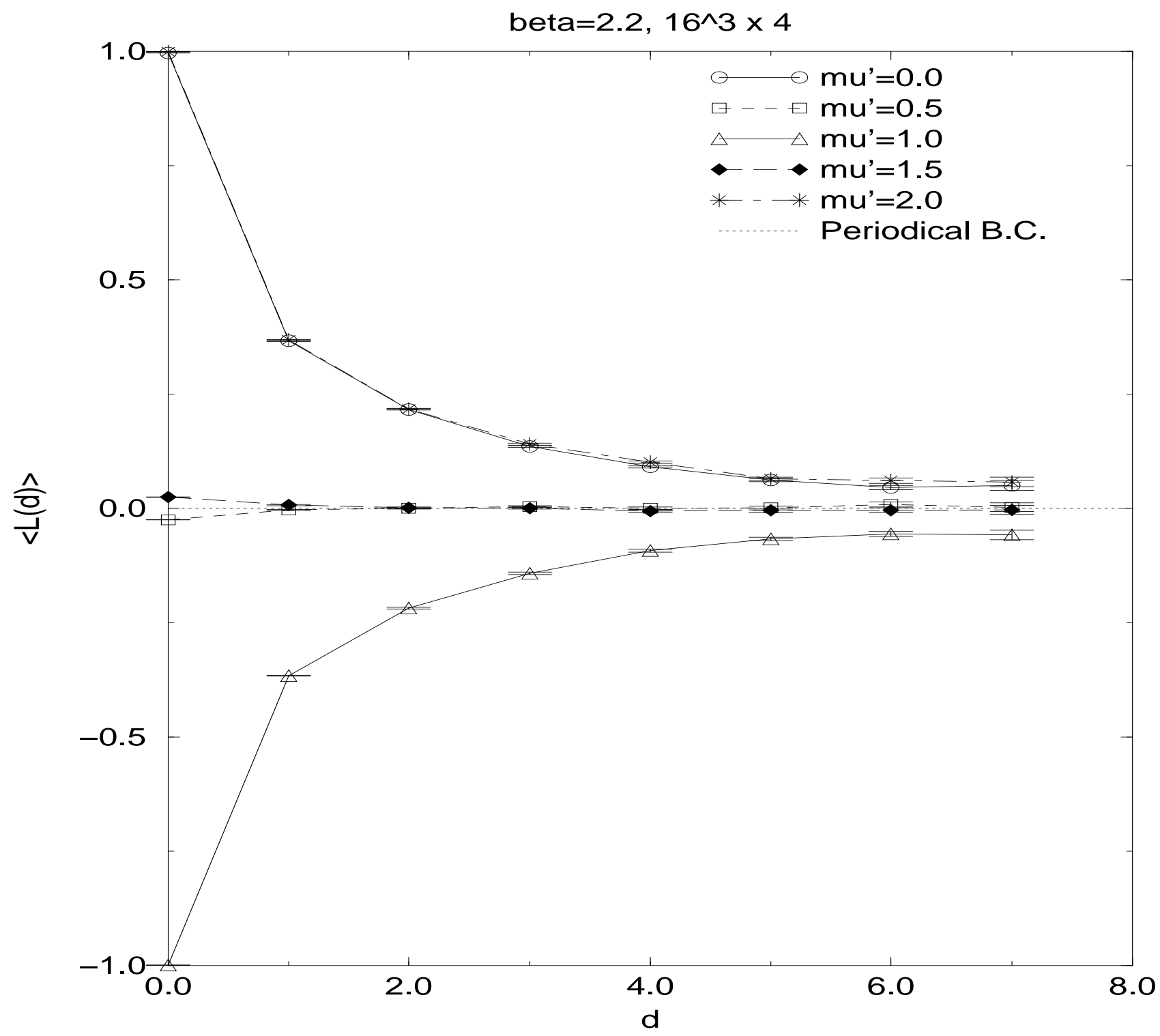

Figure 6: Average Polyakov line operator for $\beta=2.2$ (confinement phase) computed for different boundary conditions at all lattice sites with distance to the boundary $d$. For comparison we show the result obtained with periodic boundary conditions (dotted lines), too. 


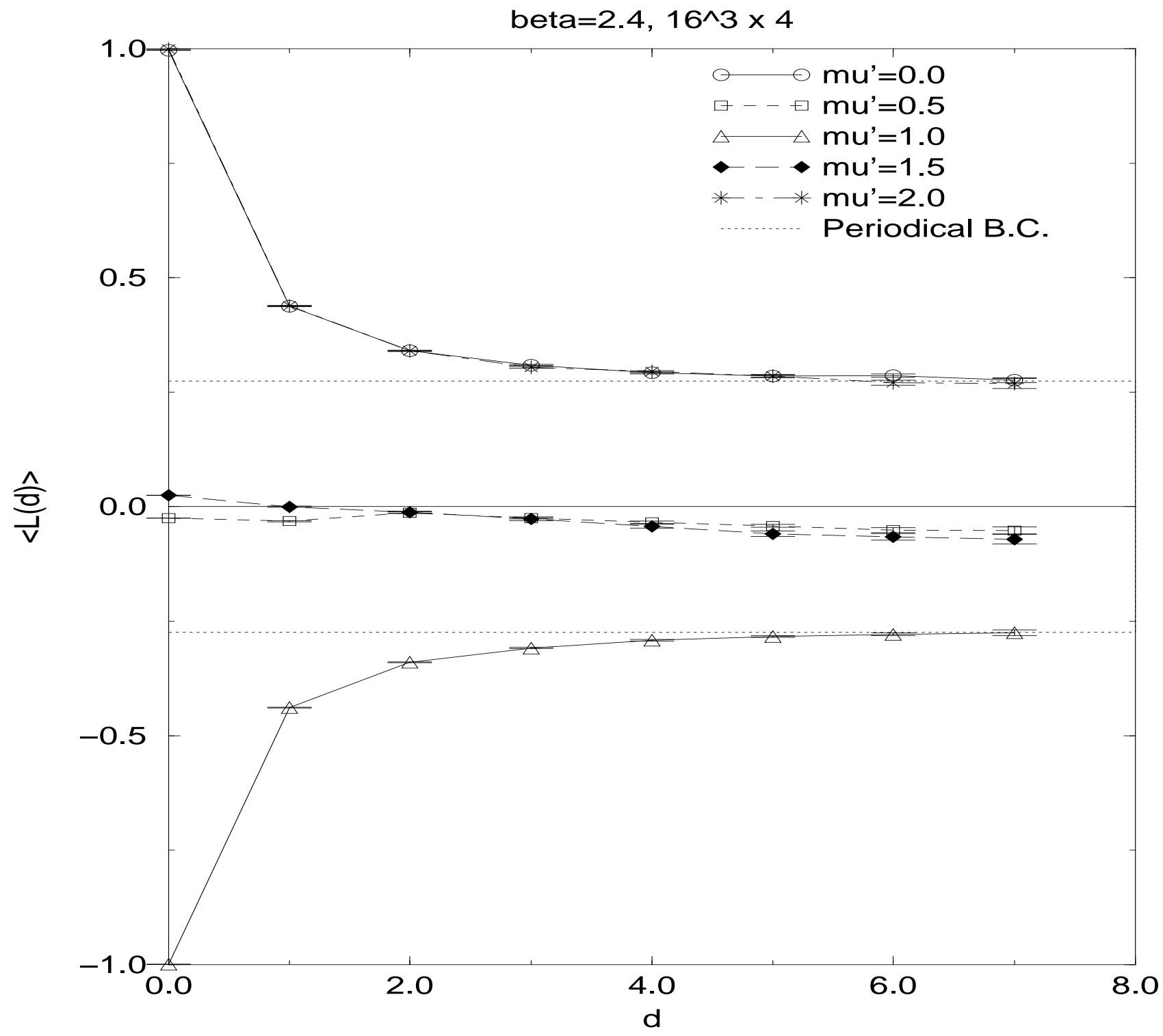

Figure 7: As in Fig. 6, but for $\beta=2.4$ (deconfinement phase). The results obtained with periodic boundary conditions were splitted into the two $Z(2)$ asymmetric values (dotted lines). 


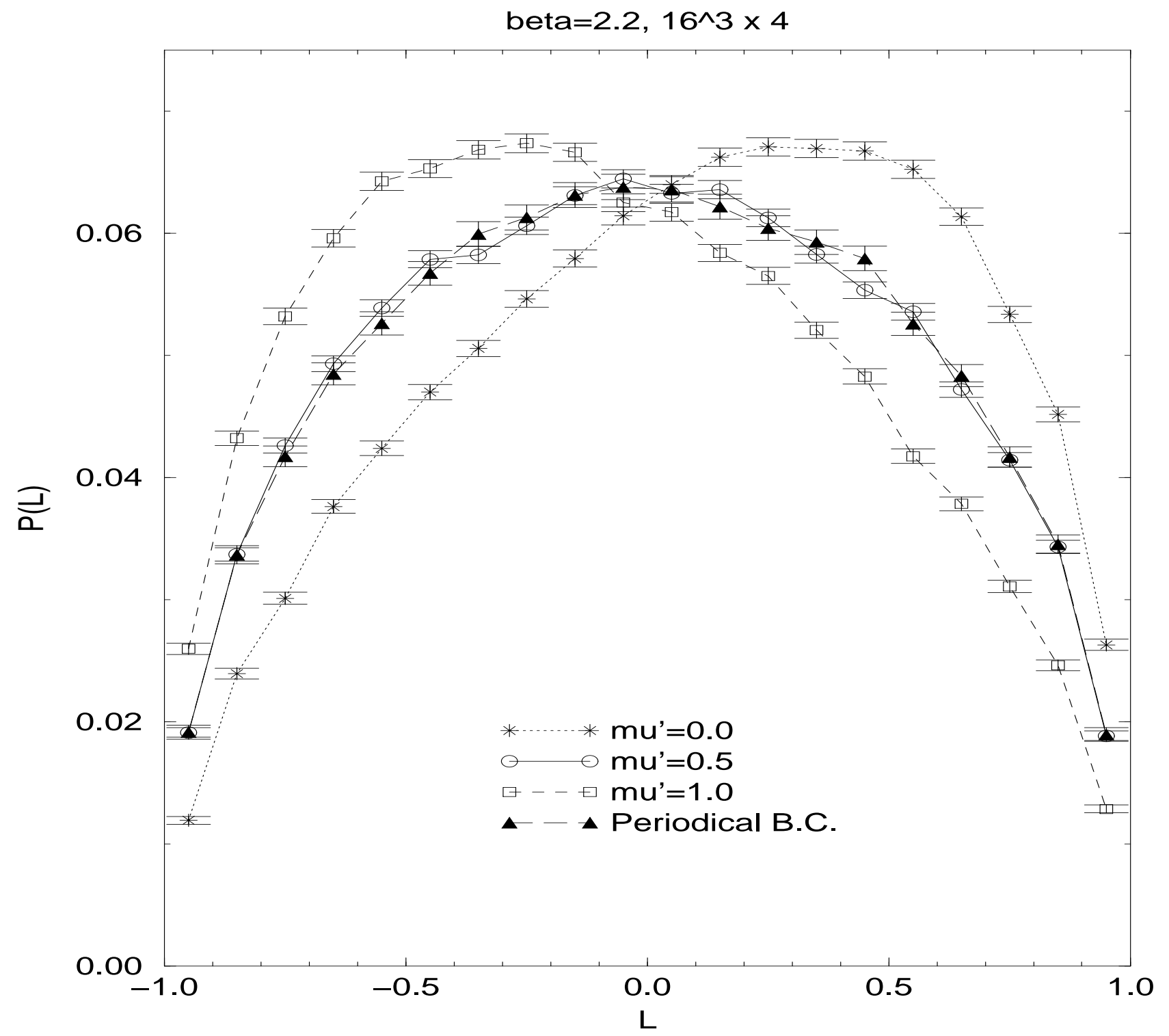

Figure 8: Distribution of local Polyakov line values for various boundary conditions (dyon case for $\mu^{\prime}=0.5$, pure magnetic monopole case $\mu^{\prime}=0.0,1.0$ ). The Polyakov line operator has been computed at all sites with distance $d=5$ from the boundary. For comparison we show also the case of periodic boundary conditions obtained at the same $\beta=2.2$. 


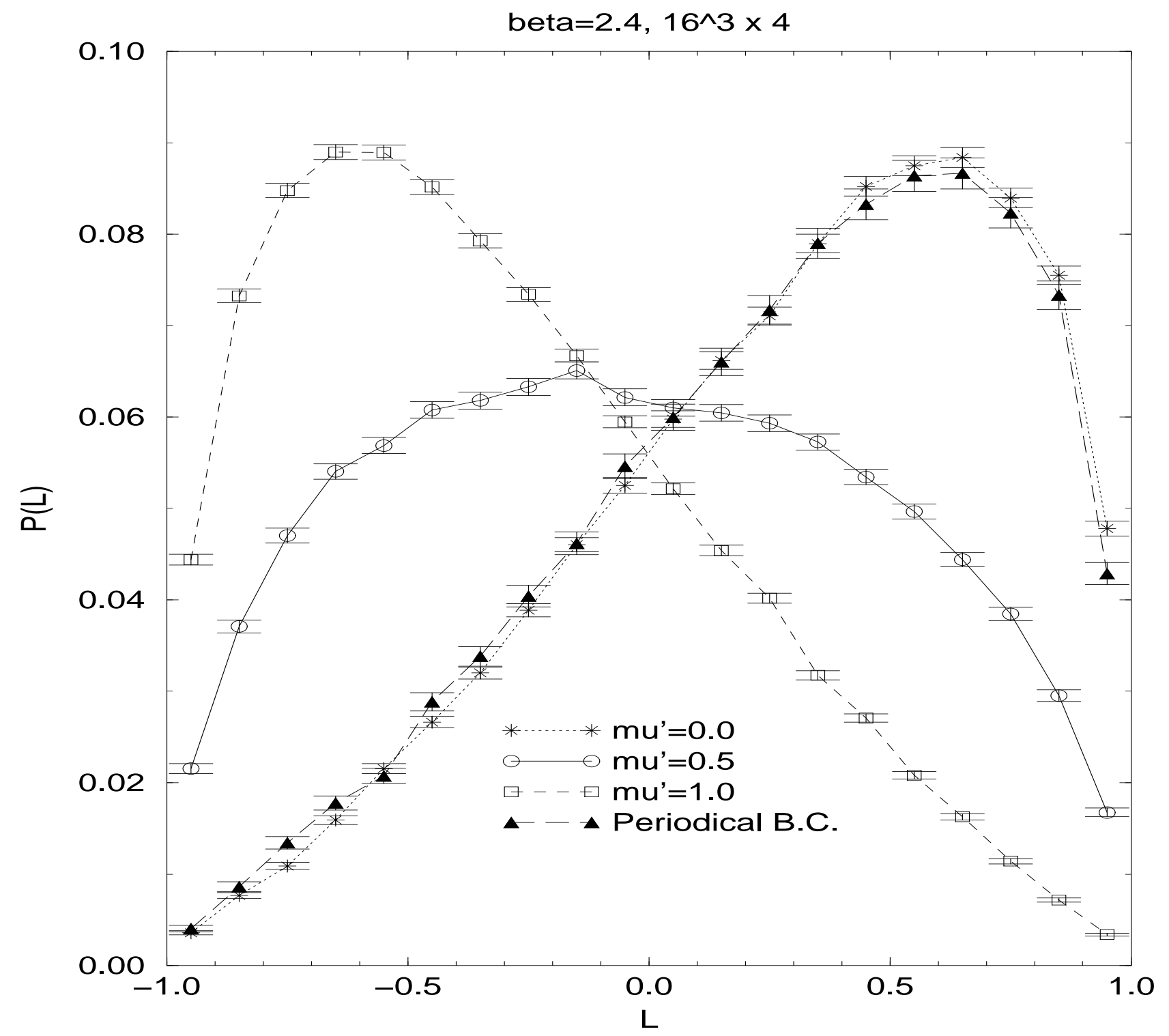

Figure 9: The same as in Fig. 8, but for $\beta=2.4$. All runs were taken with comparable statistics $(O(500)$ configurations). In the periodic boundary case, this statistics was not enough in order to observe any tunneling between the $Z(2)$ states. Therefore, the distribution occurs to be asymmetric confined to only one of the $Z(2)$ orientations. 


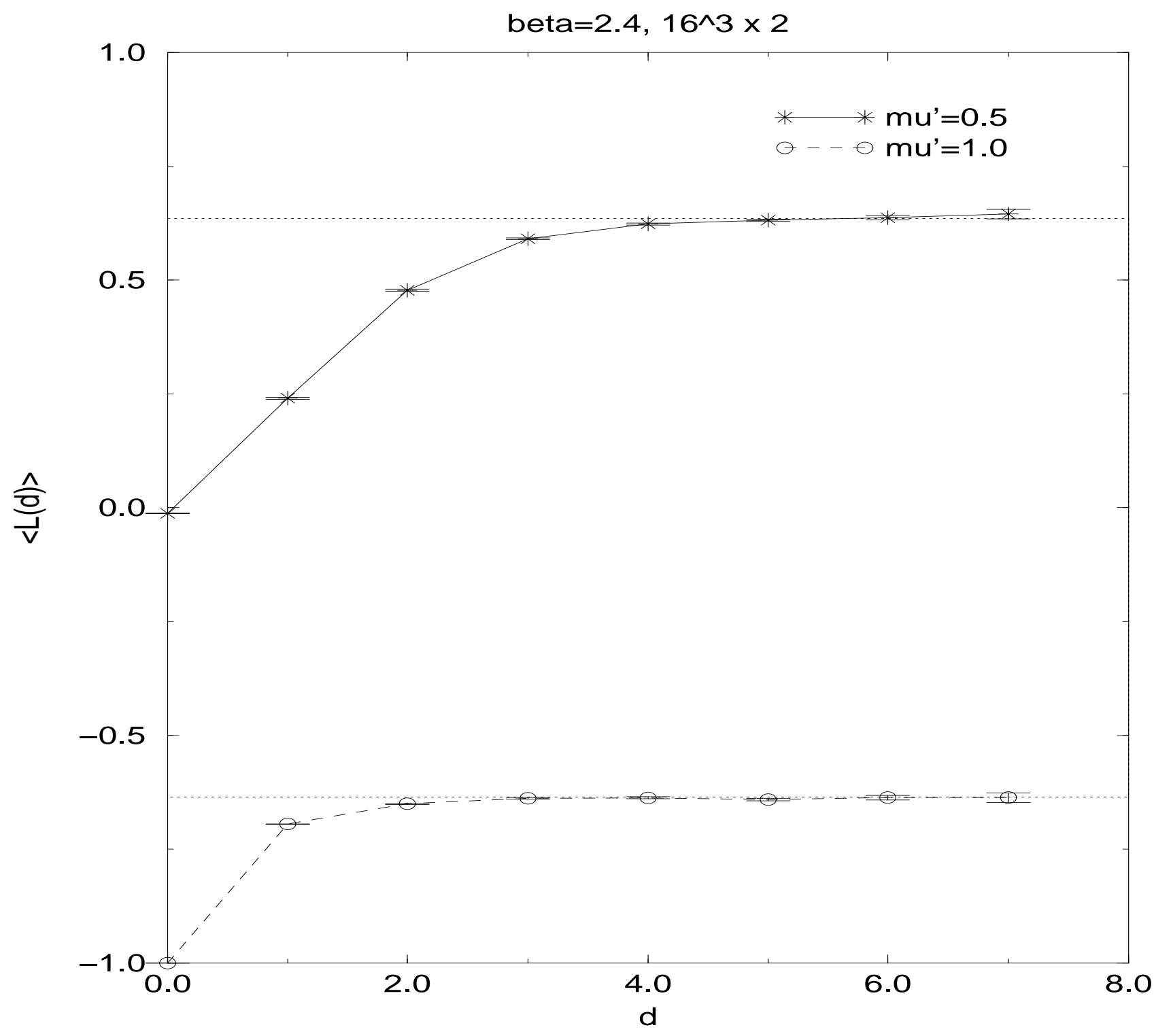

Figure 10: As for Fig. 7, but for higher temperature $N_{t}=2$. 